\title{
Appropriateness of Dropout Layers and Allocation of Their 0.5 Rates across Convolutional Neural Networks for CIFAR-10, EEACL26, and NORB Datasets
}

\author{
Vadim V. Romanuke \\ Polish Naval Academy, Gdynia, Poland
}

\begin{abstract}
A technique of DropOut for preventing overfitting of convolutional neural networks for image classification is considered in the paper. The goal is to find a rule of rationally allocating DropOut layers of 0.5 rate to maximise performance. To achieve the goal, two common network architectures are used having either 4 or 5 convolutional layers. Benchmarking is fulfilled with CIFAR-10, EEACL26, and NORB datasets. Initially, series of all admissible versions for allocation of DropOut layers are generated. After the performance against the series is evaluated, normalized and averaged, the compromising rule is found. It consists in non-compactly inserting a few DropOut layers before the last convolutional layer. It is likely that the scheme with two or more DropOut layers fits networks of many convolutional layers for image classification problems with a plenty of features. Such a scheme shall also fit simple datasets prone to overfitting. In fact, the rule "prefers" a fewer number of DropOut layers. The exemplary gain of the rule application is roughly between $10 \%$ and $50 \%$.
\end{abstract}

Keywords - CIFAR-10, convolutional neural network, DropOut, EEACL26, image classification, NORB, overfitting.

\section{INTRODUCTION}

The dropout method stylized as DropOut has been introduced to prevent overfitting [1], [2]. Modern convolutional neural networks (CNNs) widely exploit this technique [3], [4]. At each training stage, individual activations are removed with a probability. Only the reduced $\mathrm{CNN}$ is trained on the data at that stage. The removed nodes are then reinserted into the $\mathrm{CNN}$ with their original weights.

The probability that a hidden node will be dropped in the training stages is usually 0.5 . No substantiations for this value have been suggested since 2012 [2], [4], [5]. It is intuitively supposed that for input nodes the drop probability should be less than 0.5 , because information is directly lost when input nodes are ignored.

Allocation of DropOut layers (DOLs) is another open question, even bigger than that of the probability. Again, it is naively believed that a DOL should be allocated after each convolutional layer (ConvL). However, no reasonable confirmations standing for that paradigm are known [6], [7]. Diverse allocations of DOLs are not proved as well.

\section{BACKGROUND}

A typical example of overfitting is when a $\mathrm{CNN}$ performs poorly on held-out test data because it was trained on a small training set. As shown in [2], overfitting is greatly reduced by randomly omitting half of the feature detectors on each training case. Each neuron learns to detect a feature that is generally helpful for producing the correct answer by preventing complex feature co-adaptations. Along with overfitting decrement, DropOut significantly improves the training speed owing to avoiding the training of all nodes [8]. However, the total duration of training may become longer.

The central idea of DropOut is to take a large model that overfits easily and repeatedly the sample and train smaller sub-models from it [8]. The essential drawback of DropOut is that it increases training time. A CNN with DOLs typically is trained longer than the same CNN without them [5], [9], [10]. This is caused by the fact that the parameter updates are very noisy. In each training case, a different random CNN architecture is also effectively trained.

Given a definite image classification problem (ICP), the crucial question is how many DOLs are needed. Obviously, not every ICP needs a DOL after each ConvL [4], [11], [12]. This is influenced by a number of classes. Complexity and diversity of images to be classified influence as well. Eventually, it is not certain whether the allocation of DOLs may have its own "DropOut-Out" when a CNN having DOLs contains at least one ConvL which is not followed by a DOL.

\section{GOAL OF THE RESEARCH}

Despite the prevalence of DOLs, finding a rule of their rational allocation is needed. Rationality herein will use the performance criterion. The goal of the research is to find a rule that would suggest the number and positions of DOLs maximising the CNN performance. A possible influence of the main parameters of an ICP, which are the image size and number of image categories, is to be ascertained. To achieve the goal, the following tasks are to be fulfilled:

1. To select a benchmark ICP.

2. To generate a series of all admissible versions for DOL allocation.

3. To evaluate performance against the DOL allocation series.

4. To find a version of DOL allocation that maximises the performance.

5. To link the result of the performance evaluation to the rule of rationally allocating DOLs.

\section{ICP TO BE BENCHMARKED}

Let a common CNN architecture be defined for allocating DOLs in all admissible versions. The definition implies a constant number of ConvLs $N_{\text {ConvL }}$. Most medium-sized ICPs are solvable with $\mathrm{CNNs}$ having 4 or 5 ConvLs. Thus, the common extensible CNN architecture is either 
$C_{1} \rightarrow R_{1} \rightarrow P_{1} \rightarrow C_{2} \rightarrow R_{2} \rightarrow P_{2} \rightarrow C_{3} \rightarrow R_{3} \rightarrow C_{4} \rightarrow S$

or

$$
\begin{aligned}
C_{1} \rightarrow R_{1} \rightarrow P_{1} \rightarrow & C_{2} \rightarrow R_{2} \rightarrow P_{2} \rightarrow C_{3} \rightarrow R_{3} \rightarrow P_{3} \rightarrow \\
& \rightarrow C_{4} \rightarrow C_{5} \rightarrow S
\end{aligned}
$$

by ConvLs $\left\{C_{i}\right\}_{i=1}^{N_{\text {conl }}}$, three ReLUs $\left\{R_{j}\right\}_{j=1}^{3}$, maximum pooling layers (MPLs) $\left\{P_{i}\right\}_{i=1}^{N_{\text {conn }}-2}$, and a softmax layer (SML) $S$. Numbers of filters in the first three ConvLs by $N_{\text {ConvL }}=4$ are 20,50,100. Numbers of filters in the first four ConvLs by $N_{\text {ConvL }}=5$ are 20, 50,100, 200. The last ConvL has a number of filters equal to a number of image categories. MPLs are of the standard $2 \times 2$ window with the stride of 2 and without zero-padding. Both architectures (1) and (2) will be tested, by varying the number of DOLs and their allocations. A single DOL (of 0.5 rate) can be inserted right after a ConvL.

An ICP to be benchmarked is the CIFAR-10 dataset. The dataset consists of 60000 colour images divided into 50000 images intended for training and 10000 images intended for testing (validating). The original CIFAR-10 dataset image is of size $32 \times 32 \times 3$, represented as $32 \times 32$ matrix in each of the three colour channels. Although the CIFAR-10 dataset has only 10 image categories (6000 images per category), its images are heterogeneous and miscellaneous (Fig. 1) diversely representing such classes as "airplane", "automobile", "bird", "cat", "deer", "dog", "frog", "horse", "ship", "truck" [2], [10], [13], [14]. The diversity of the CIFAR-10 entries is expected to ensure good generalization of the rule of rationally allocating DOLs.
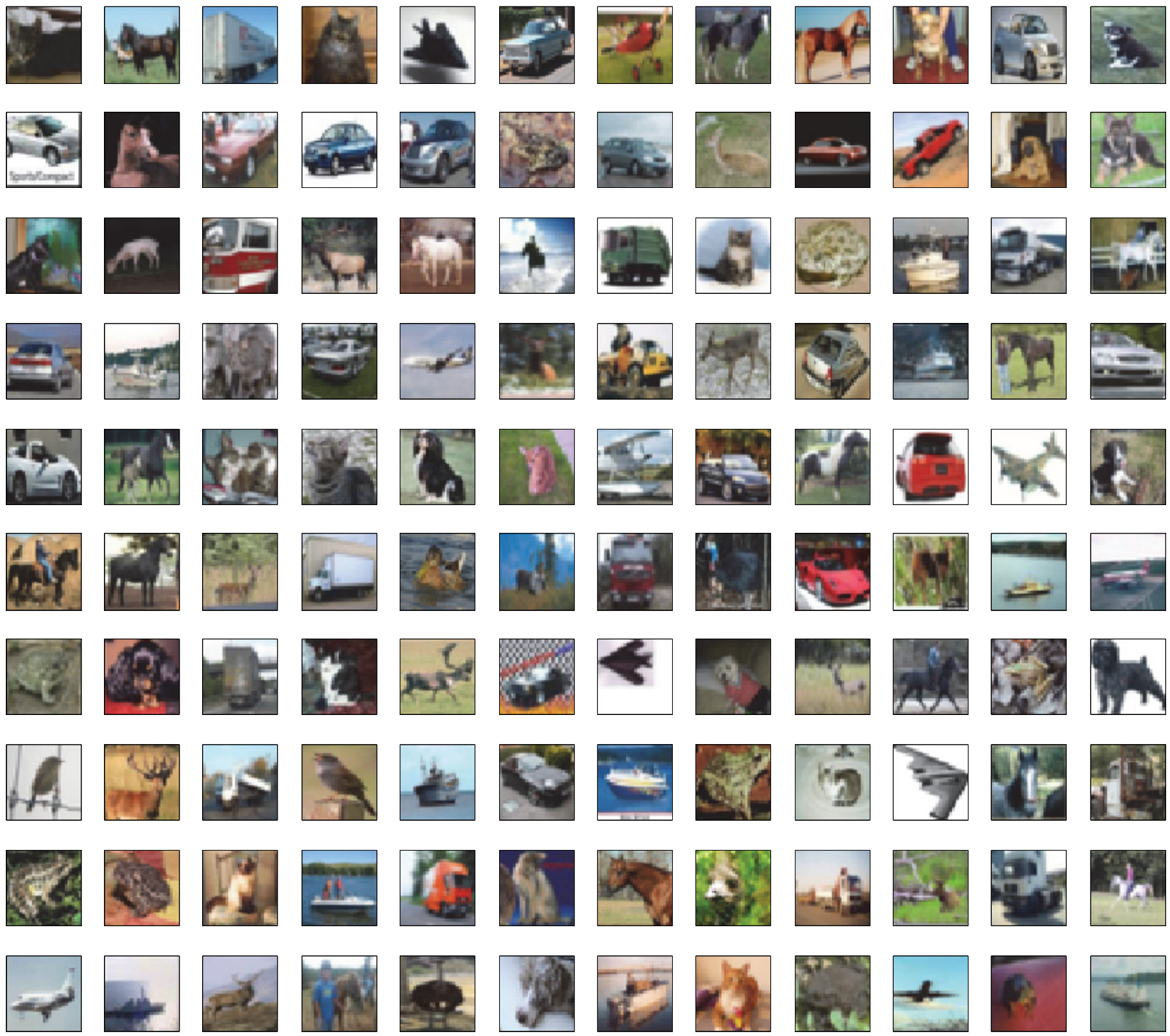

Fig. 1. Heterogeneousness and diversity of the CIFAR-10 entries in their 10 image categories. The background is diverse being either natural or artificial. The images are so tiny that, for the human eye, it takes rather a long period to conclude on some of them (e. g., try the fourth and fifth images in the bottom line from the left side). The CIFAR-10 entries vary from simpler images having a monotonous background (with an abrupt transition) to multicoloured images. 
In addition to testing two CNNs (1) and (2) with DOLs, the image size will be increased from 32 with a step of 4 up to 64 . Let the EEACL26 and NORB datasets (Fig. 2 and Fig. 3) be used [13], [15], [16] along with CIFAR-10, with resizing their images to the same range. This should allow noticing the differentiation of $\mathrm{CNN}$ performance against the DOL allocation series. Thus, those allocations, where the performance is close-to-best, will be seen.

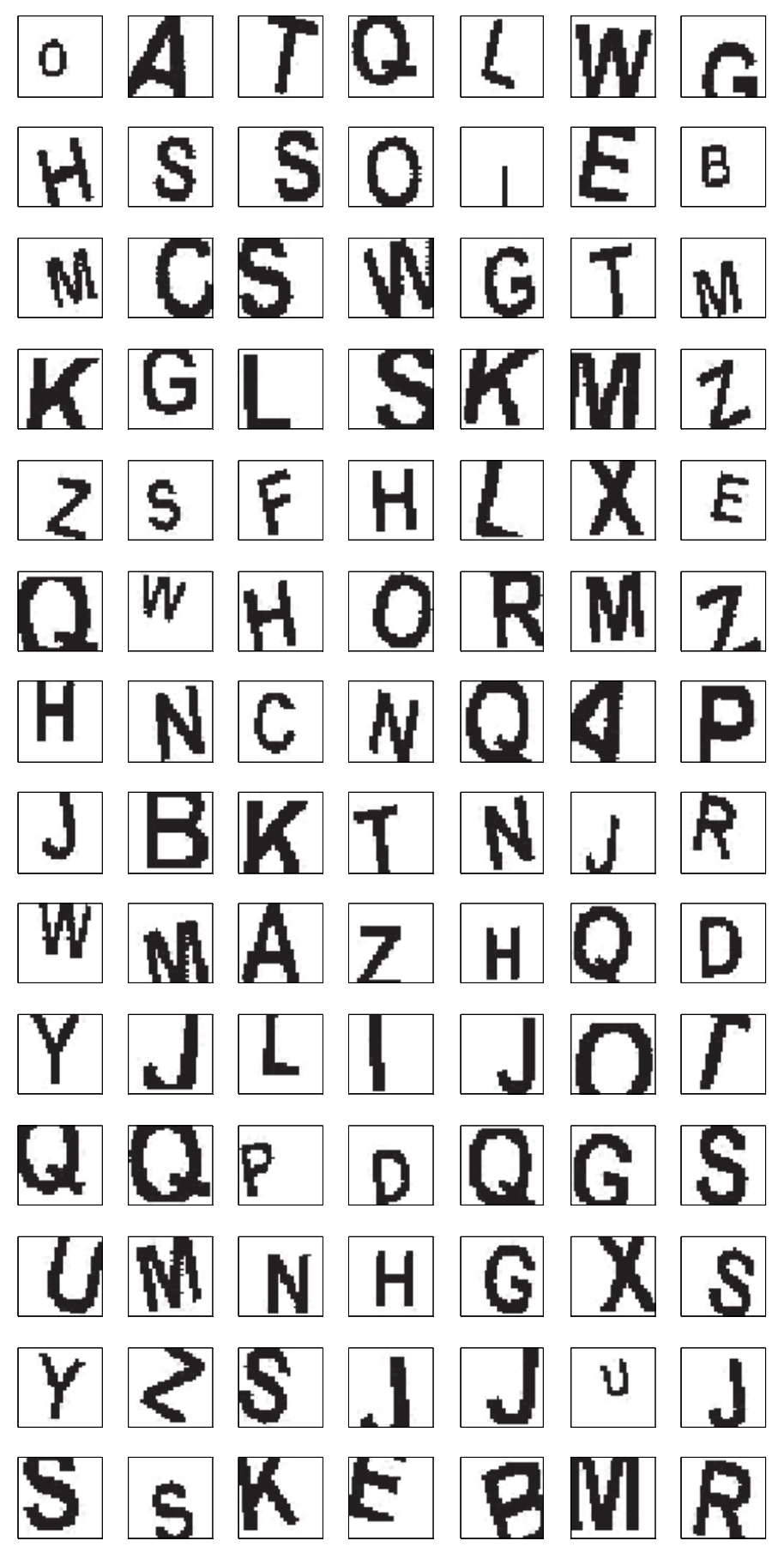

Fig. 2. Less heterogeneousness of the EEACL26 entries in their 26 image categories representing enlarged capital letters of the English alphabet [15]. The background is constantly white, although some grayish pieces appear after downsampling from the original image. Entries of this dataset have all main geometrical attributes of a dataset of real-world images - lines, curves, circles, crosses, roughness, etc. Like entries of CIFAR-10 and NORB, the letters are distorted with shifting, scaling, skewing (rotations), and trimming.
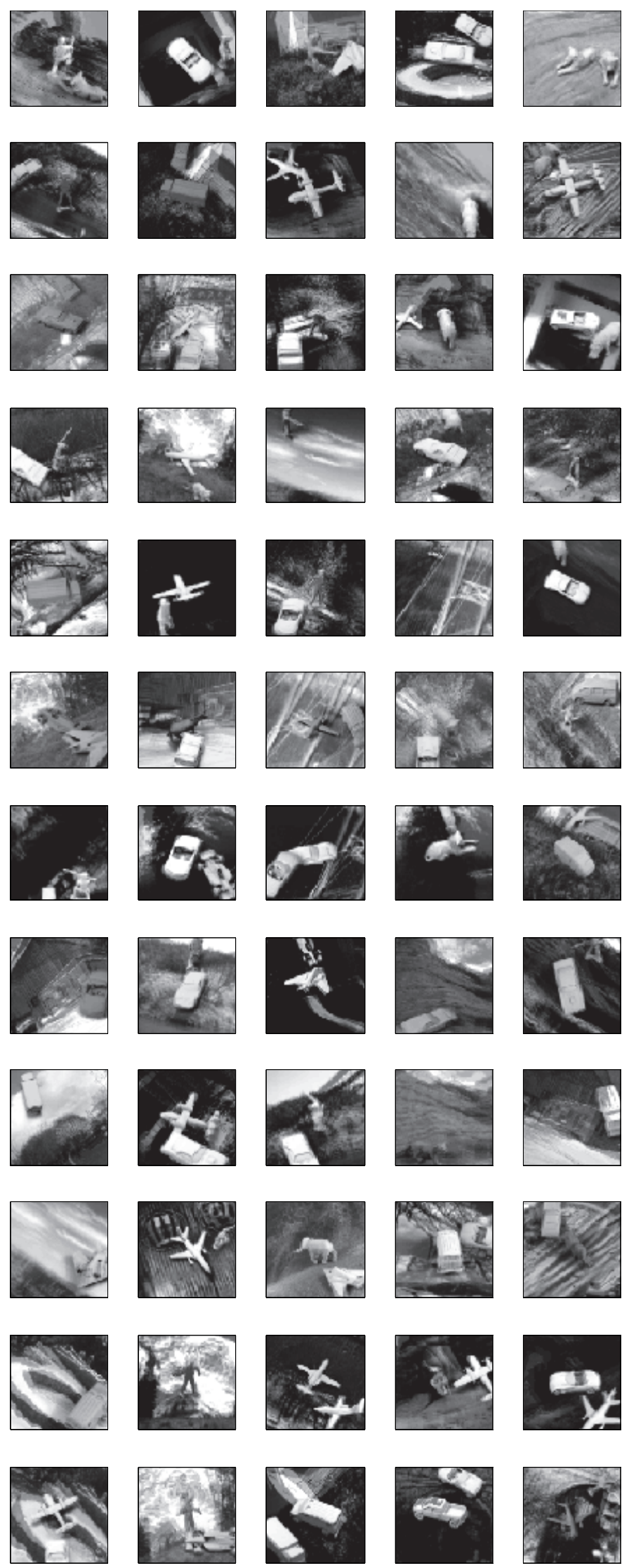

Fig. 3. The original NORB dataset contains grayscale 8 -bit $108 \times 108 \times 2$ images of 50 toys belonging to 5 generic categories (four-legged animals, human figures, airplanes, trucks, and cars), where the 6-th image category of the blank image is appended. The human eye can hardly recognise animals and human figures. Cars and trucks are almost undistinguishable. 
The datasets CIFAR-10, EEACL26, and NORB intended to be benchmarked are heterogeneous themselves. CIFAR-10 is of natural images, and entries of the EEACL26 dataset are artificial. NORB contains toy images which are losslessdownsampled to $32 \times 32 \times 2$. Entries of NORB are not so diverse than those of CIFAR-10, and there are only 6 image categories against 10 in CIFAR-10. However, the diversity of the EEACL26 entries is the poorest, despite the fact that the number of EEACL26 image categories is 2.6 times greater than that of CIFAR-10, and is 4-with-a-third times greater than that of NORB. Nevertheless, the dataset EEACL26 is scalable, and as many EEACL26 entries can be generated as needed.

\section{SERIES OF All AdMisSible VERSIONS FOR DOL ALLOCATION}

Owing to that a ConvL is either followed or not followed with a DOL, a series of all admissible versions for DOL allocation is generated by binary numbers. Maximal number of DOLs is $N_{\text {ConvL }}$. For CNN (1), there are $2^{4}=16$ versions for DOL allocation. For CNN (2), there are $2^{5}=32$ versions for DOL allocation. Each version corresponds to a 4-digit or 5 -digit binary number, respectively, where its $i$-th position from the left indicates whether a DOL is inserted after the $i$-th ConvL (binary 1) or not (binary 0 ). Let zero correspond to an architecture without DOLs. Let an architecture with a maximal number of DOLs be represented with a binary number having only ones. Thus, the $k$-th admissible version for DOL allocation corresponds to the binary number $k-1$ represented with $N_{\text {ConvL }}$ positions by $k=\overline{1,2^{N_{\text {Conv }}}}$. The second version corresponds to either $0001_{(2)}$ or $00001_{(2)}$ implying a CNN with a single DOL inserted after the last ConvL (before an SML). The last but one version corresponds to either $1110_{(2)}$ or $11110_{(2)}$ implying that DOLs are inserted after each ConvL, except the last ConvL.

\section{Evaluation of Performance Against THE DOL ALLOCATION SERIES}

To notice the differentiation of $\mathrm{CNN}$ performance, a suitable number of epochs for evaluation is 8 . Performance is evaluated at each $p$-th epoch by $p=\overline{1,8}$ under three parameters: image size $H \times H$ (in every colour channel for CIFAR-10 and NORB), $N_{\text {ConvL }}$ and the $k$-th version for DOL allocation, where

$$
H \in S_{\text {image }}=\{32,36,40,44,48,52,56,60,64\} .
$$

Denoting the error rate after the $p$-th epoch by $v_{p}^{\langle\mathrm{ICP}\rangle}$ for an ICP, the 8-dimensional vectors

$$
\mathbf{V}_{\mathrm{CIFAR}}\left(H, N_{\mathrm{ConvL}}, k\right)=\left[v_{p}^{\langle\mathrm{CIFAR}\rangle}\left(H, N_{\mathrm{ConvL}}, k\right)\right]_{1 \times 8},
$$

and

$$
\mathbf{V}_{\text {EeACL26 }}\left(H, N_{\text {ConvL }}, k\right)=\left[v_{p}^{\langle\text {EEACL26 }}\left(H, N_{\text {ConvL }}, k\right)\right]_{1 \times 8},
$$

and

$$
\mathbf{V}_{\mathrm{NORB}}\left(H, N_{\mathrm{ConvL}}, k\right)=\left[v_{p}^{\langle\mathrm{NORB}\rangle}\left(H, N_{\mathrm{ConvL}}, k\right)\right]_{1 \times 8},
$$

are to be evaluated for the three ICPs, by $N_{\text {ConvL }} \in\{4,5\}$ and (3) at $k=\overline{1,2^{N_{\text {ConvL }}}}$.

The absolute error rates (4)-(6) are incomparable because of the heterogeneousness of the three datasets. The normalized averages

$$
\tilde{v}^{\langle\mathrm{ICP}\rangle}\left(H, N_{\mathrm{ConvL}}, k\right)=\frac{\sum_{p=1}^{8} v_{p}^{\langle\mathrm{ICP}\rangle}\left(H, N_{\mathrm{ConvL}}, k\right)}{\underline{\max _{q=1,2^{N \mathrm{Conv}}}} \sum_{p=1}^{8} v_{p}^{\langle\mathrm{ICP}\rangle}\left(H, N_{\mathrm{Conv}}, q\right)}
$$

are comparable along the $k$ axis. Similarly, the normalized final-epoch performance

$$
\tilde{v}_{8}^{\langle\mathrm{ICP}\rangle}\left(H, N_{\mathrm{ConvL}}, k\right)=\frac{v_{8}^{\langle\mathrm{ICP}\rangle}\left(H, N_{\mathrm{ConvL}}, k\right)}{\underline{\max _{q=1,2^{N} \mathrm{ConvL}}} \nu_{8}^{\langle\mathrm{ICP}\rangle}\left(H, N_{\mathrm{ConvL}}, q\right)}
$$

serves for comparison. However, the image size can be canceled via simple averaging of (7) and (8):

$$
\tilde{\tilde{v}}^{\langle\mathrm{ICP}\rangle}\left(N_{\mathrm{ConvL}}, k\right)=\frac{1}{9} \sum_{H \in S_{\text {image }}} \tilde{v}^{\langle\mathrm{ICP}\rangle}\left(H, N_{\mathrm{ConvL}}, k\right)
$$

and

$$
\tilde{\tilde{v}}_{8}^{\langle\mathrm{ICP}\rangle}\left(N_{\mathrm{ConvL}}, k\right)=\frac{1}{9} \sum_{H \in S_{\text {image }}} \tilde{v}_{8}^{\langle\mathrm{ICP}\rangle}\left(H, N_{\mathrm{ConvL}}, k\right)
$$

for respective ICPs.

Figures 4-9 show evaluation of 12 polylines (9) and (10) for CIFAR-10, EEACL26, and NORB datasets. There are two main variants of minimum points: $k=3$ (6 times of 12) and $k=5$ (3 times). Minima at $k=1, k=7$, and $k=11$ happened to appear just once. The average of polylines (9) in Figs. 4, 6 and 8 shown in Fig. 10 confirms that a CNN with a single DOL inserted before the last ConvL, i.e., corresponding to $k=3$, which is $0010_{(2)}$ or $00010_{(2)}$, performs the best. The minimum point of polylines (10) in Figs. 4, 6 and 8 shown in Fig. 10 is achieved at $k=5$, although its minimum value is very close to the value achieved at $k=3$. The averages of polylines (9) and (10) in Fig. 5, 7 and 9 shown in Fig. 11 confirm that inserting a single DOL before the last ConvL is the best practice. It is worth noting that here final-epoch performance is close to the best at $k=11$ which is $01010_{(2)}$ (caused by the influence of an unexpected result in Fig. 9). 


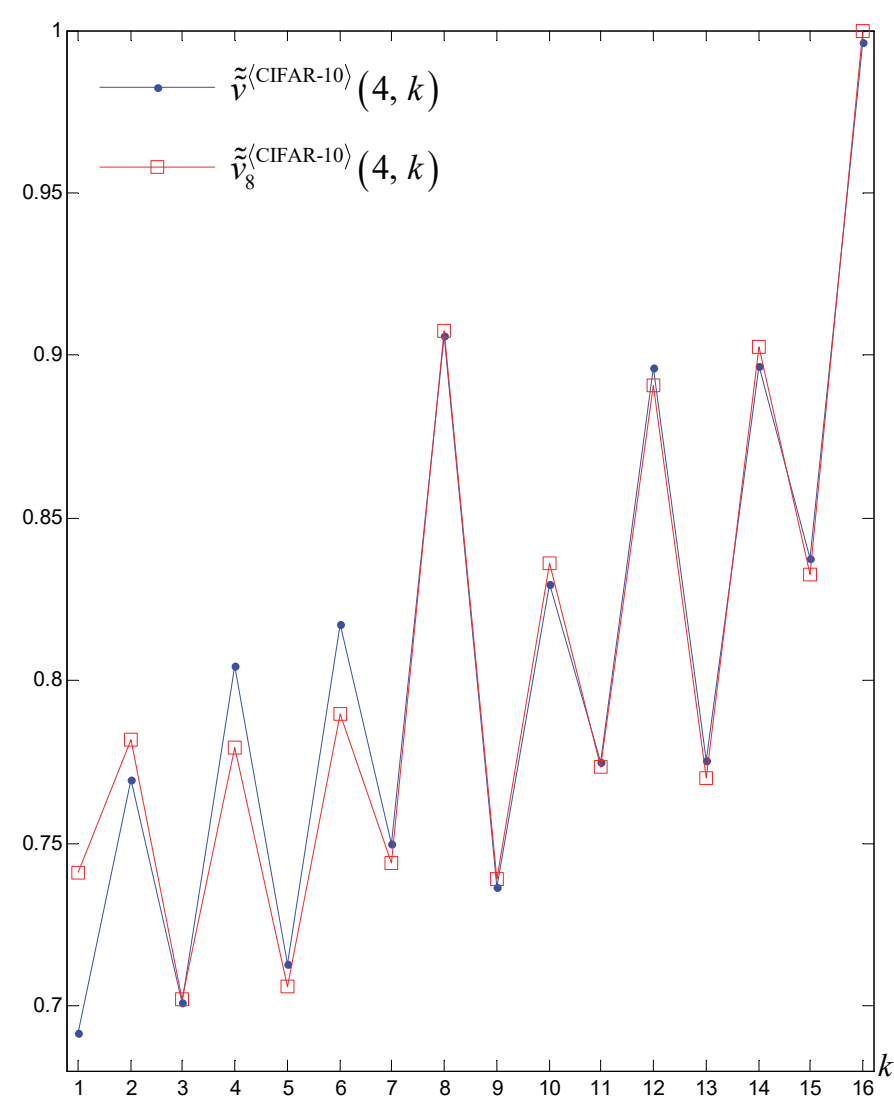

Fig. 4. Performance of CNNs with 4 ConvLs on CIFAR-10. Minimum points of these polylines are different $(k=1$ and $k=3)$, but close to each other.

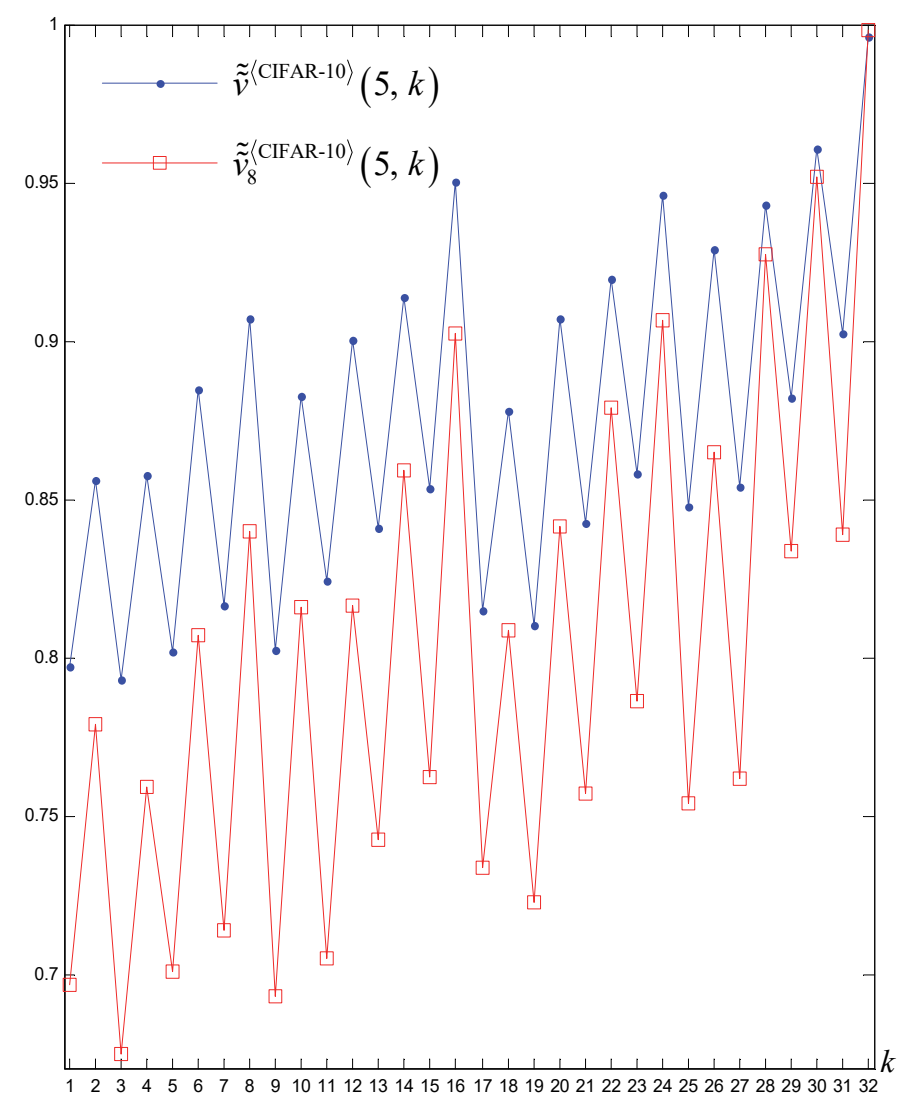

Fig. 5. Performance of CNNs with 5 ConvLs on CIFAR-10. Minima coincide corresponding to $00010_{(2)}$. The peaks at $k=8$ and $k=16$ remind those ones in Fig. 4. Inserting all possible 5 DOLs at once is the worst version similarly.

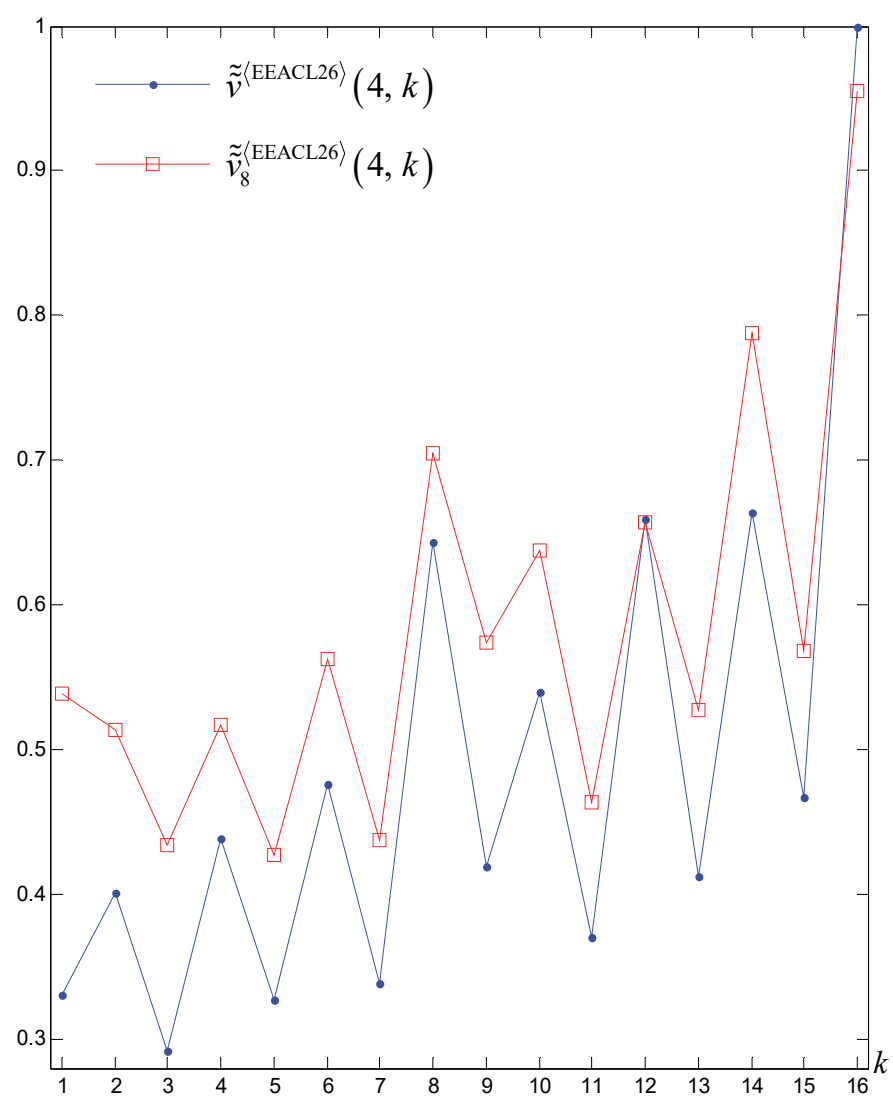

Fig. 6. Performance of CNNs with 4 ConvLs on EEACL26. Minimum points of these polylines are different $(k=3$ and $k=5)$, but still close to each other. The minimum of (9) is undisguised now. The peaks repeat themselves.

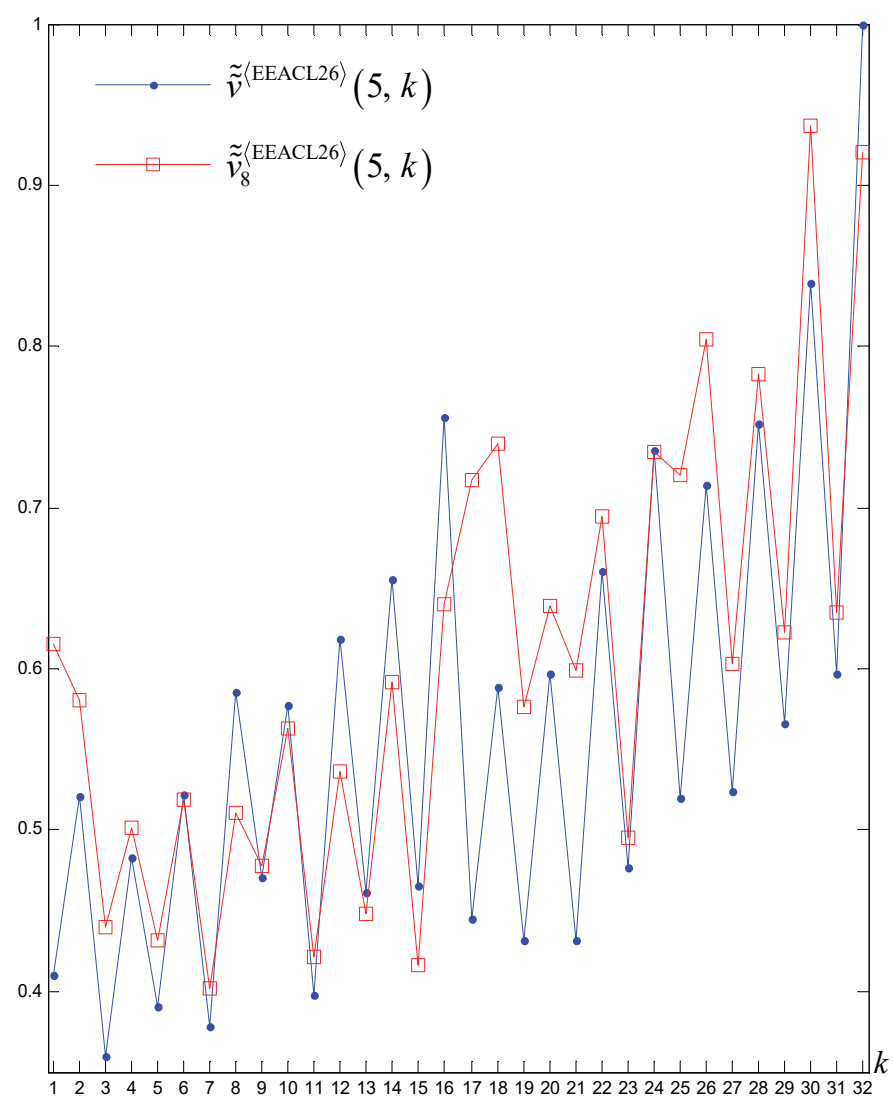

Fig. 7. Performance of CNNs with 5 ConvLs on EEACL26. The minimum of (9) is the same $(k=3)$. The minimum of $(10)$ is moved to the right $(k=7)$. 


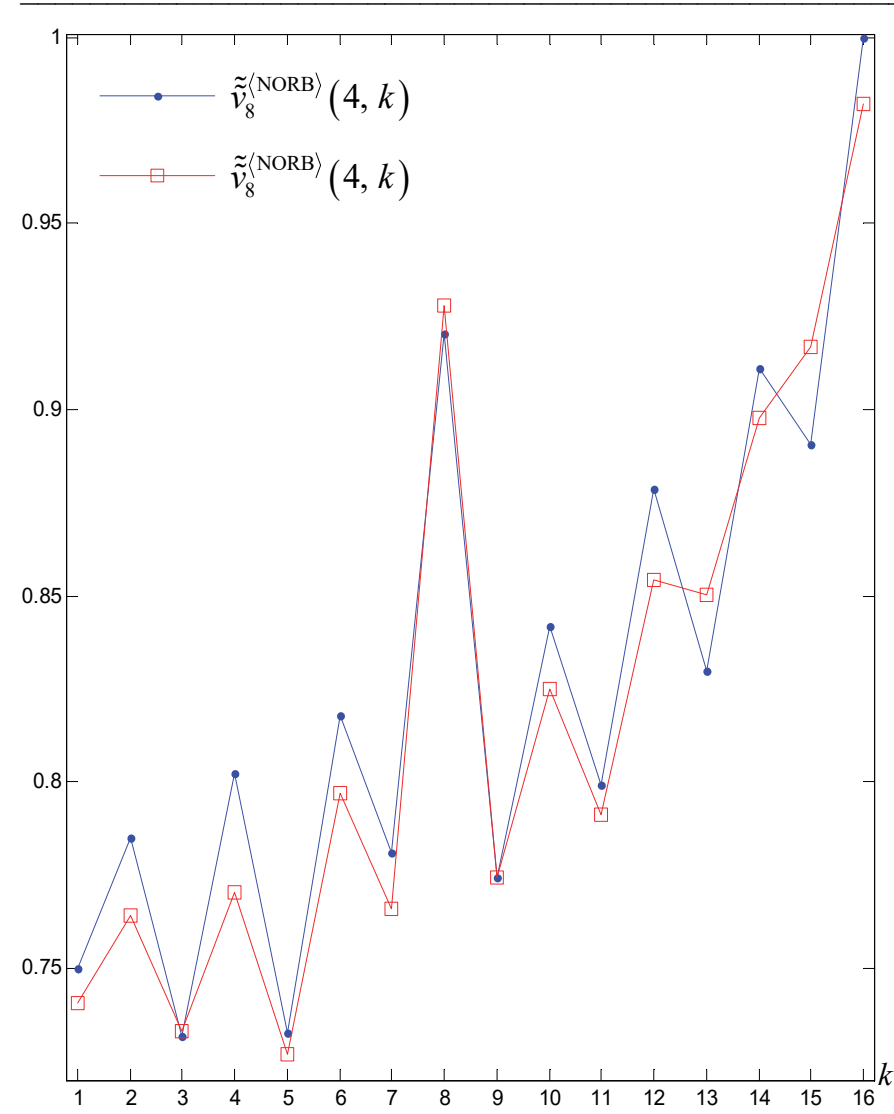

Fig. 8. Performance of CNNs with 4 ConvLs on NORB. Minima coincide corresponding, strangely enough, to $0100_{(2)}$. The peak at $k=8$ is obvious.

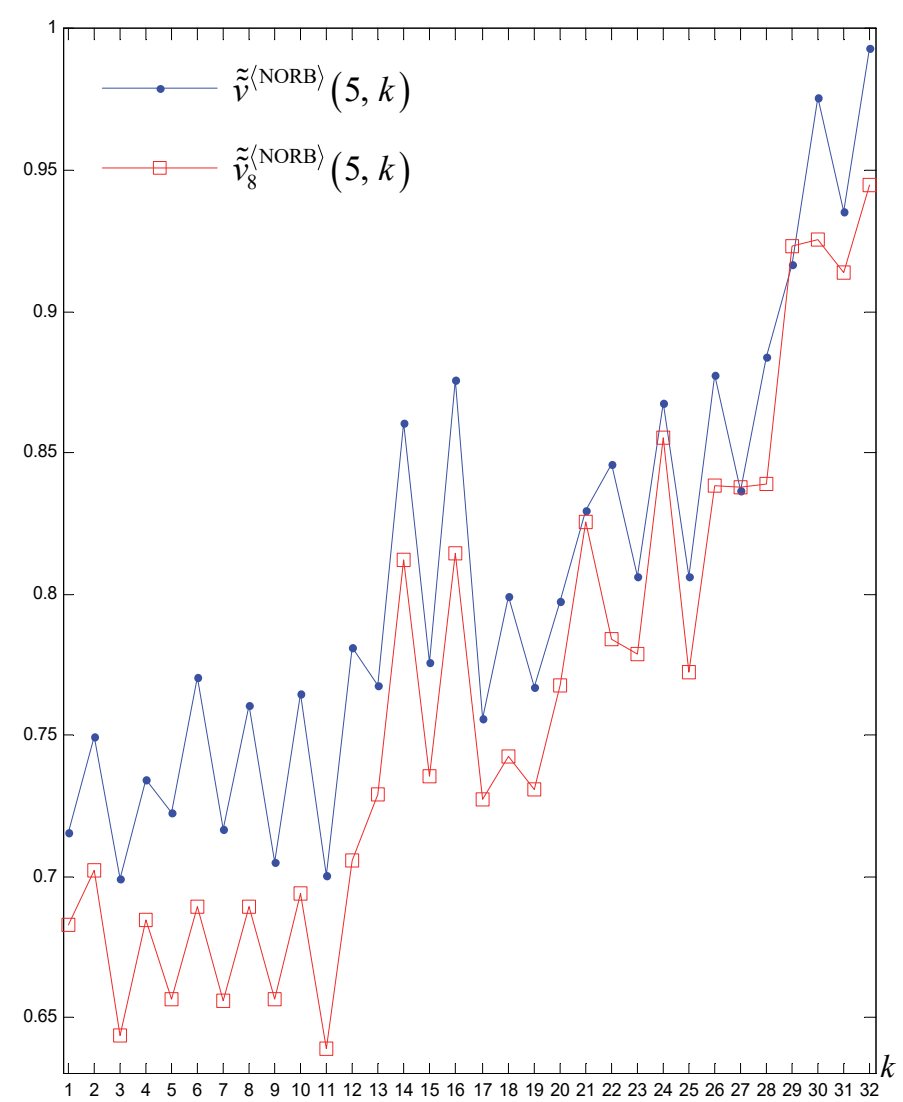

Fig. 9. Performance of CNNs with 5 ConvLs on NORB. Minima that correspond to $00010_{(2)}$ and $01010_{(2)}$ are statistically different, but very close.

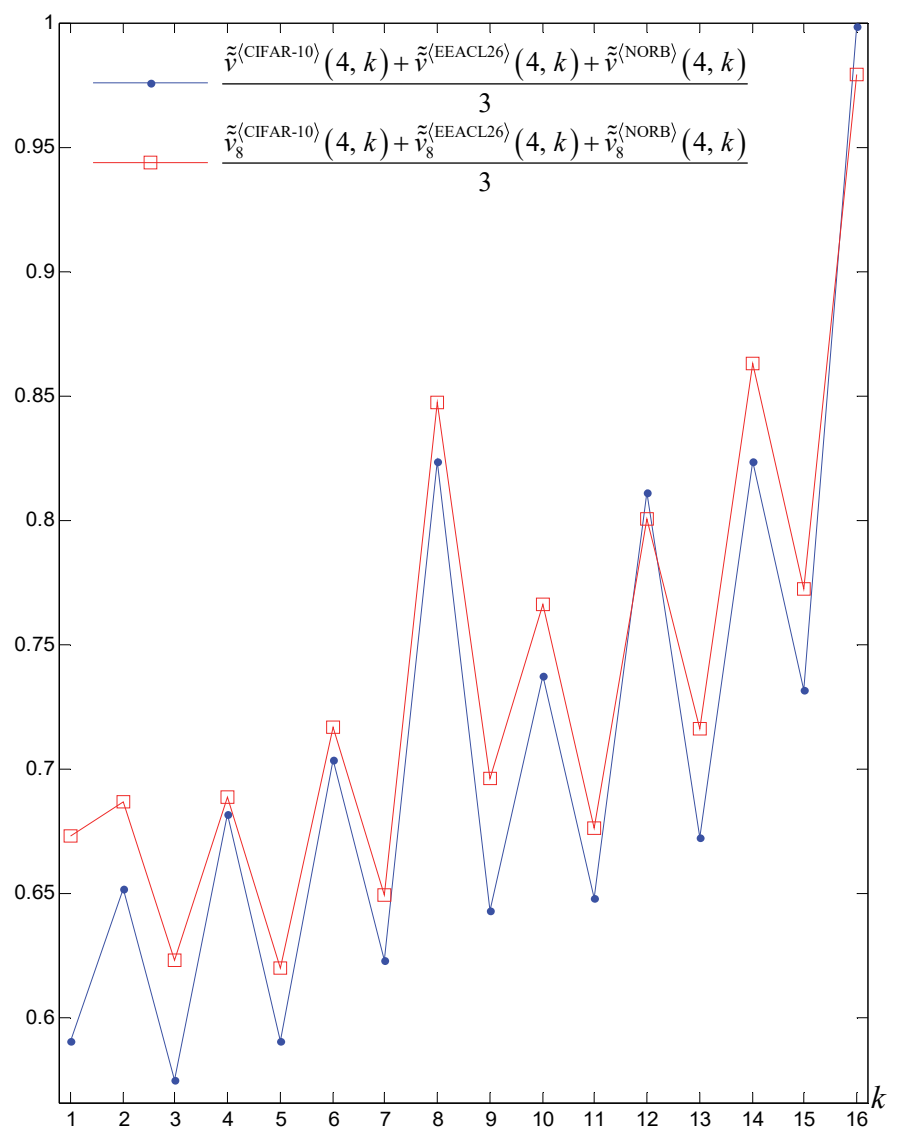

Fig. 10. The averaged performance by $N_{\text {ConvL }}=4$. Minima do not coincide.

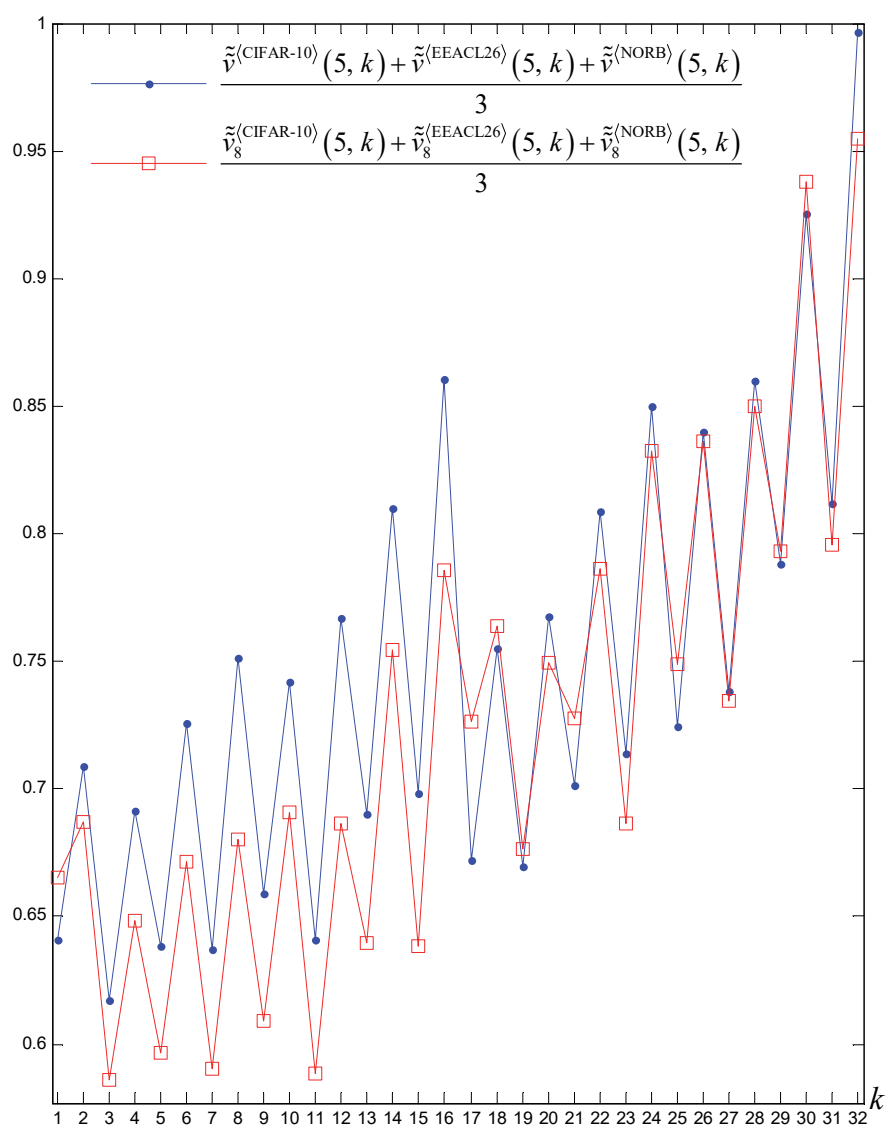

Fig. 11. The averaged performance by $N_{\text {ConvL }}=5$. Minima coincide at $k=3$, although final-epoch performance is close to the best at $k=11$ (see Fig. 9). 
Another aspect is how an error rate relatively descends. Although the starting performance of CNNs with many DOLs is worse than that with a few DOLs or without them, DOLs may accelerate convergence. The relative error rate descent (RERD) is

$$
g_{p}^{\langle\mathrm{IICP}\rangle}\left(H, N_{\mathrm{ConvL}}, k\right)=\frac{v_{p}^{\langle\mathrm{ICP}\rangle}\left(H, N_{\text {ConvL }}, k\right)}{v_{p+1}^{\langle\mathrm{IIP}\rangle}\left(H, N_{\mathrm{ConvL}}, k\right)} \text { by } p=\overline{1,7}
$$

The final-epoch RERD

$$
g_{1-8}^{\langle\text {IIC }\rangle}\left(H, N_{\text {ConvL }}, k\right)=\frac{v_{1}^{\langle\text {ICP }\rangle}\left(H, N_{\text {ConvL }}, k\right)}{v_{8}^{\langle\text {IICP }}\left(H, N_{\text {ConvL }}, k\right)}
$$

shall show an impact of DOLs. Obviously, RERDs (11) and (12) do not need normalization like (7) and (8). RERD (11) is reduced to

$$
\tilde{g}^{\langle\mathrm{ICP}\rangle}\left(H, N_{\mathrm{ConvL}}, k\right)=\sum_{p=1}^{7} g_{p}^{\langle\mathrm{ICP}\rangle}\left(H, N_{\mathrm{ConvL}}, k\right) .
$$

But the averaged RERD

$$
\tilde{\tilde{g}}^{\langle\text {IICP }}\left(N_{\text {ConvL }}, k\right)=\frac{1}{9} \sum_{H \in S_{\text {image }}} \tilde{g}^{\langle\text {ICP }\rangle}\left(H, N_{\text {ConvL }}, k\right)
$$

is incomparable to the averaged final-epoch RERD

$$
\tilde{g}_{1-8}^{\langle\mathrm{ICP}\rangle}\left(N_{\text {ConvL }}, k\right)=\frac{1}{9} \sum_{H \in S_{\text {image }}} g_{1-8}^{\langle\mathrm{ICP}\rangle}\left(H, N_{\text {ConvL }}, k\right)
$$

because of summing in (13). This is why (14) and (15) are normalized along the $k$ axis:

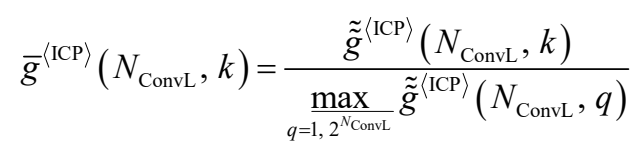

and

$$
\tilde{\tilde{g}}_{1-8}^{\langle\text {IIPP }\rangle}\left(N_{\text {ConvL }}, k\right)=\frac{\tilde{g}_{1-8}^{\langle\text {IICP }\rangle}\left(N_{\text {ConvL }}, k\right)}{\max _{q=1,2^{N \text { ConL }}} \tilde{g}_{1-8}^{\langle\text {IIP }\rangle}\left(N_{\text {ConvL }}, q\right)} .
$$

Each of the indices (16) and (17) constitutes a saw-toothed polyline (Figs. 12-17). Polylines (17) plotted for both CIFAR-10 and NORB datasets are much similar. A similarity of polylines (16) for these datasets does not raise doubts. Maximum points which indicate the best RERD coincide for CIFAR-10 and NORB, but the version $00010_{(2)}$ comes optimal only for CIFAR-10 (CNNs with 5 ConvLs). Strangely enough, the version $0011_{(2)}$ comes optimal for both CIFAR-10 and NORB (CNNs with 4 ConvLs). Optimality of the version $00101_{(2)}$ for NORB (CNNs with 5 ConvLs) is the strangest.

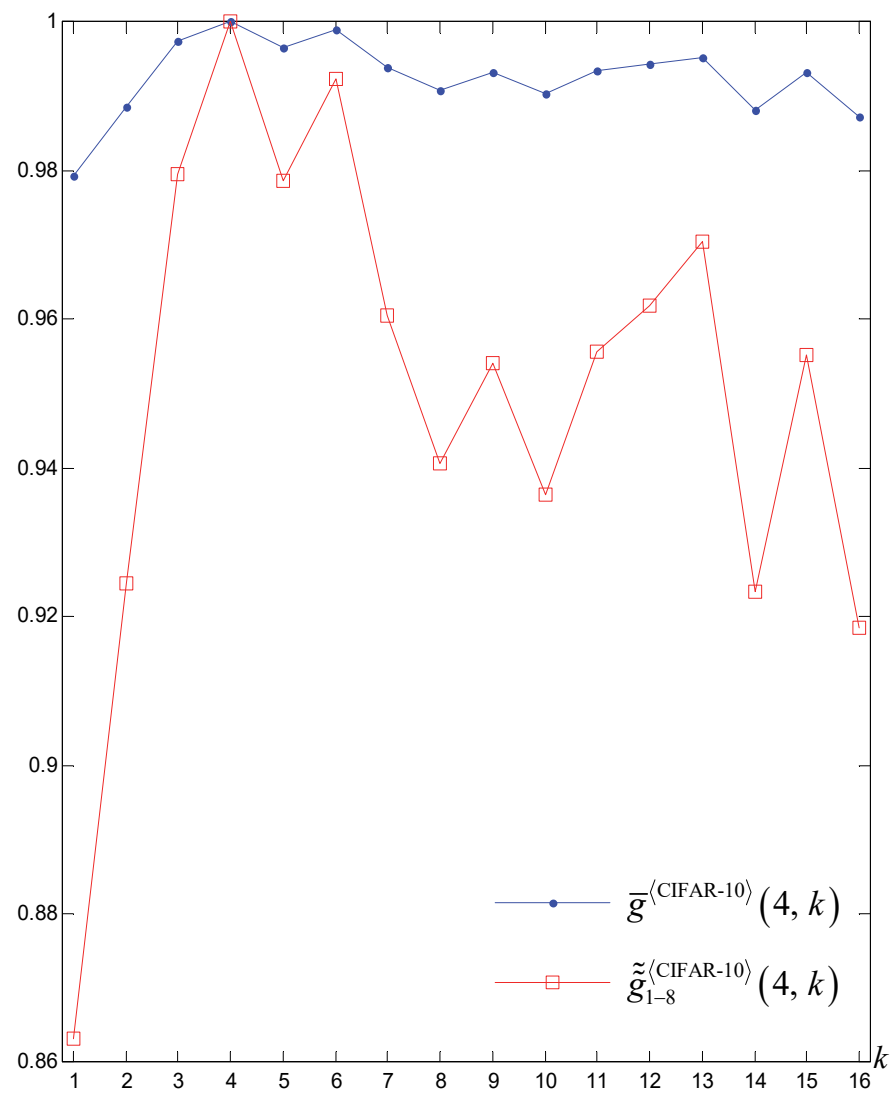

Fig. 12. The normalized averaged RERD (16) and the normalized averaged final-epoch RERD (17) by $N_{\text {ConvL }}=4$ on CIFAR-10. Maxima coincide at $k=4$.

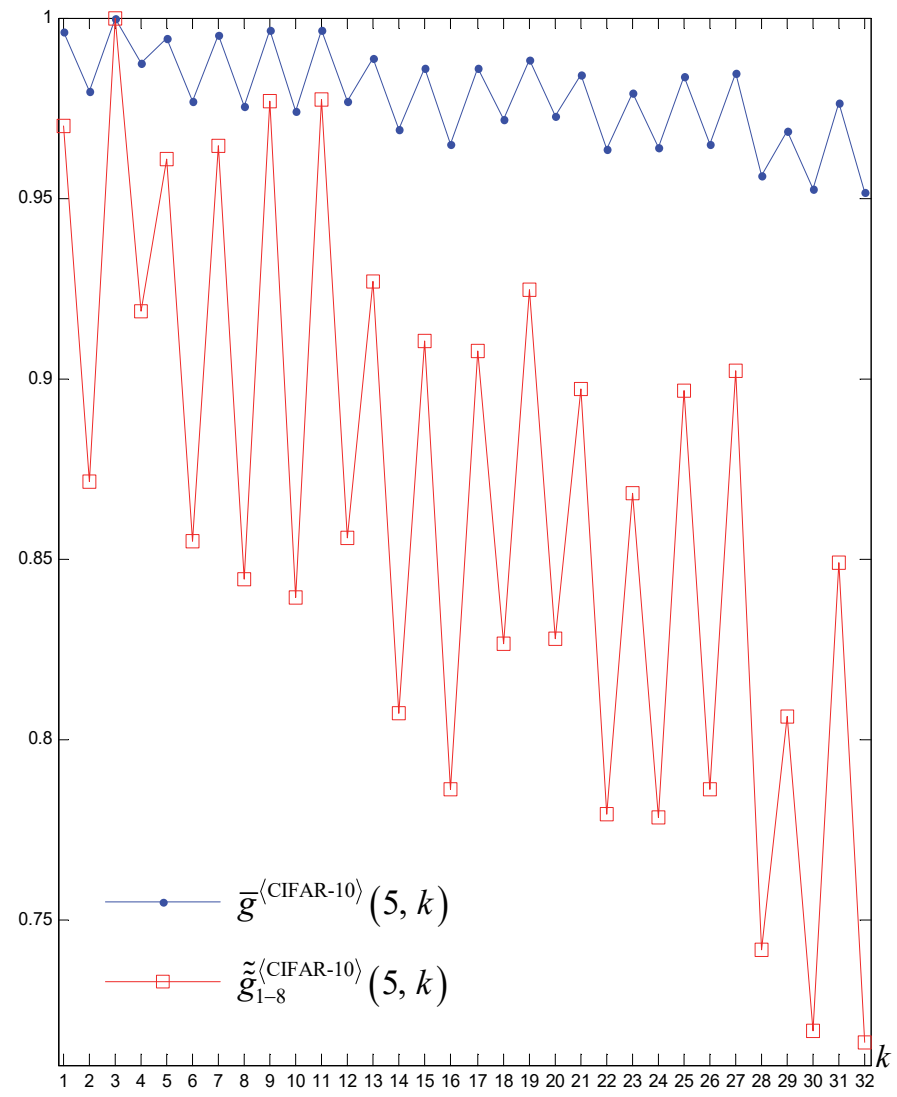

Fig. 13. The normalized averaged RERD (16) and the normalized averaged final-epoch RERD (17) by $N_{\text {ConvL }}=5$ on CIFAR-10. Maxima coincide at $k=3$, but performance of CNNs without DOLs progresses closely to them. 


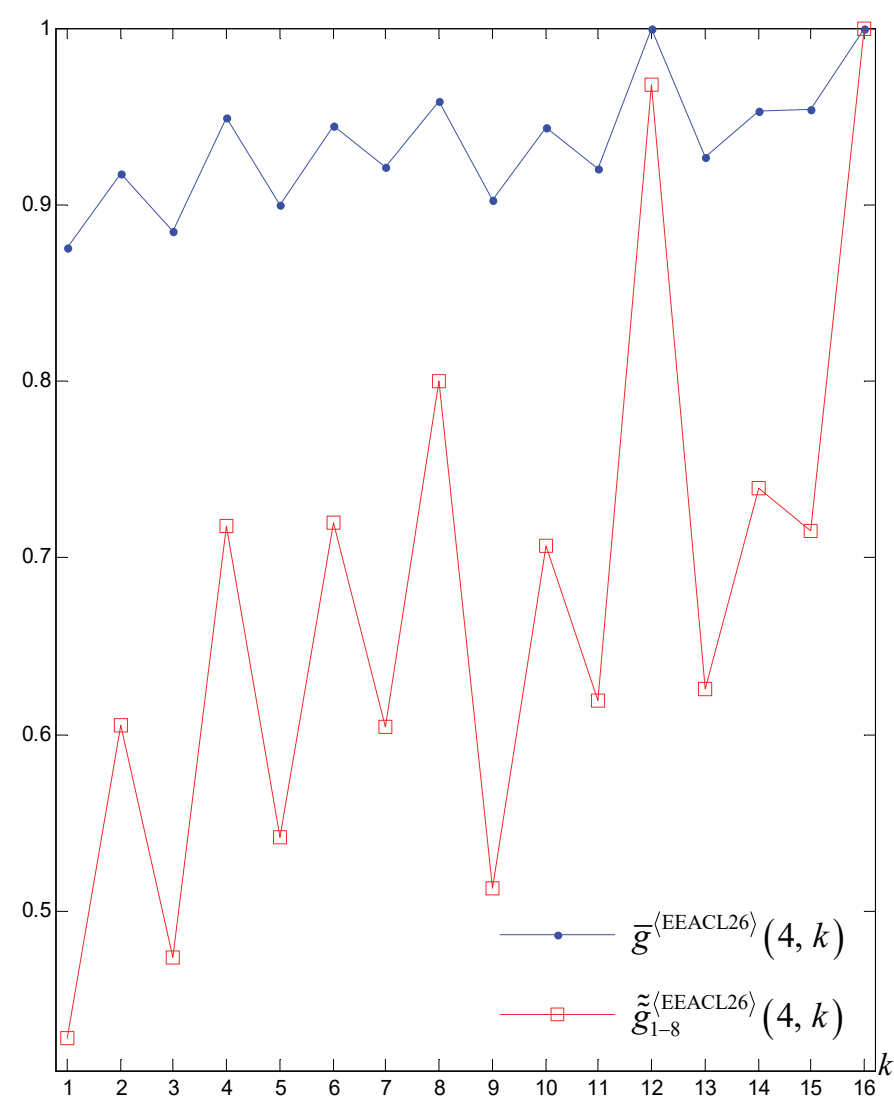

Fig. 14. The normalized averaged RERD (16) and the normalized averaged final-epoch RERD (17) by $N_{\text {ConvL }}=4$ on EEACL26. Maxima are very strange.

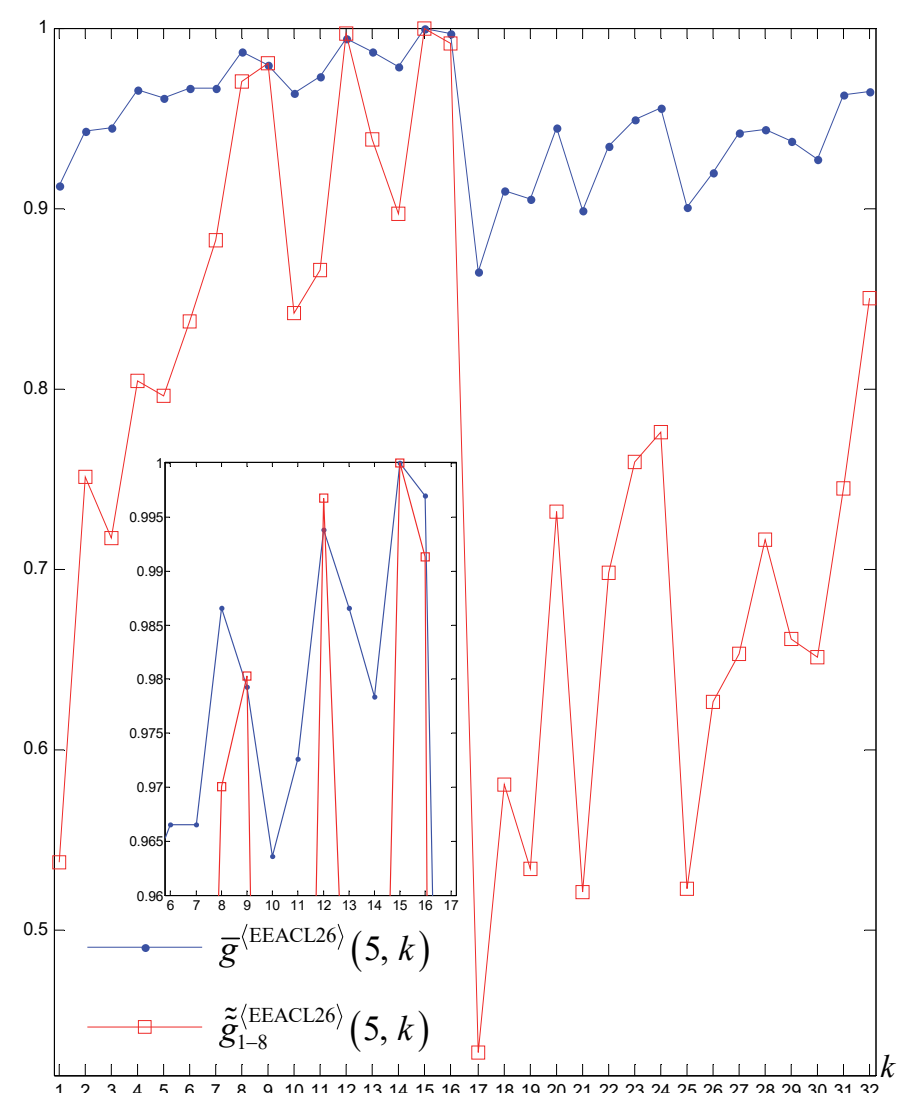

Fig. 15. The normalized averaged RERD (16) and the normalized averaged final-epoch RERD (17) by $N_{\text {ConvL }}=5$ on EEACL26. Maxima are less strange than those ones in Fig. 14, but they are pretty opposite to minima in Fig. 7.

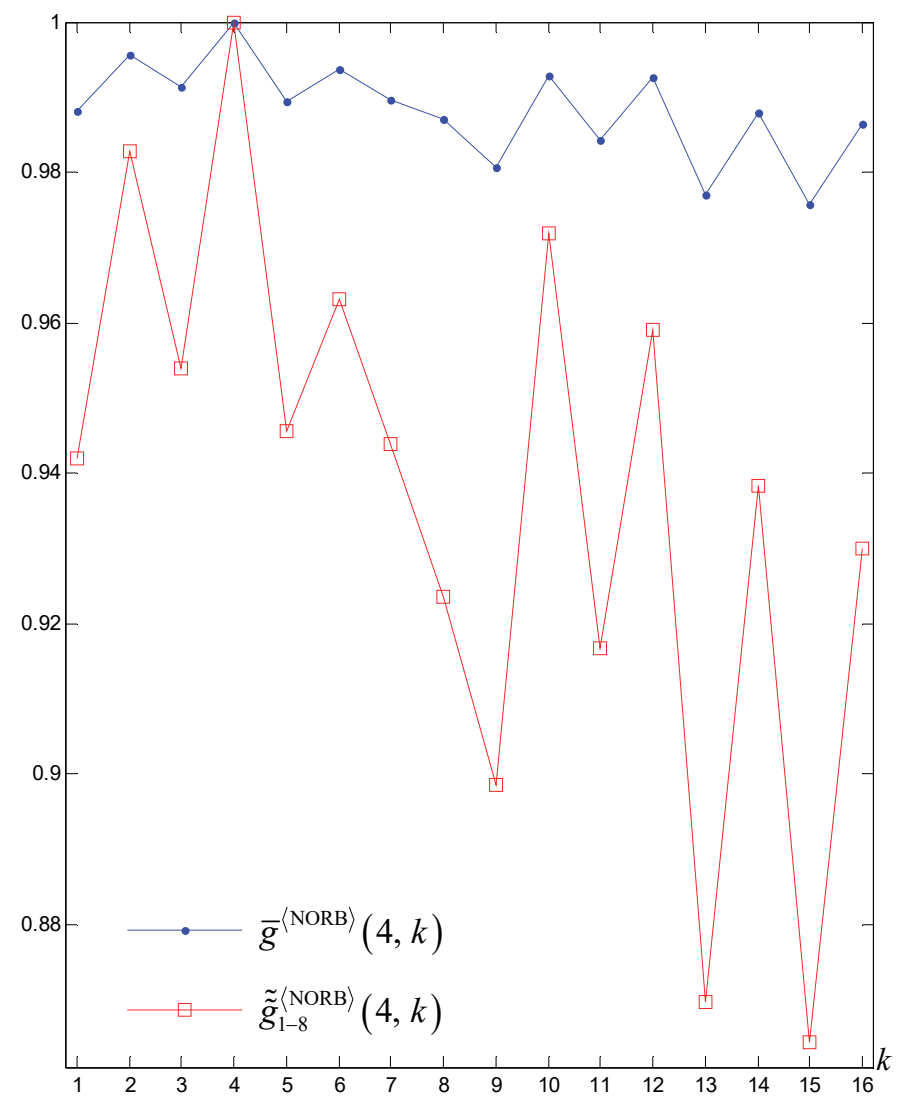

Fig. 16. The normalized averaged RERD (16) and the normalized averaged final-epoch RERD (17) by $N_{\text {ConvL }}=4$ on NORB. Maxima coincide at $k=4$.

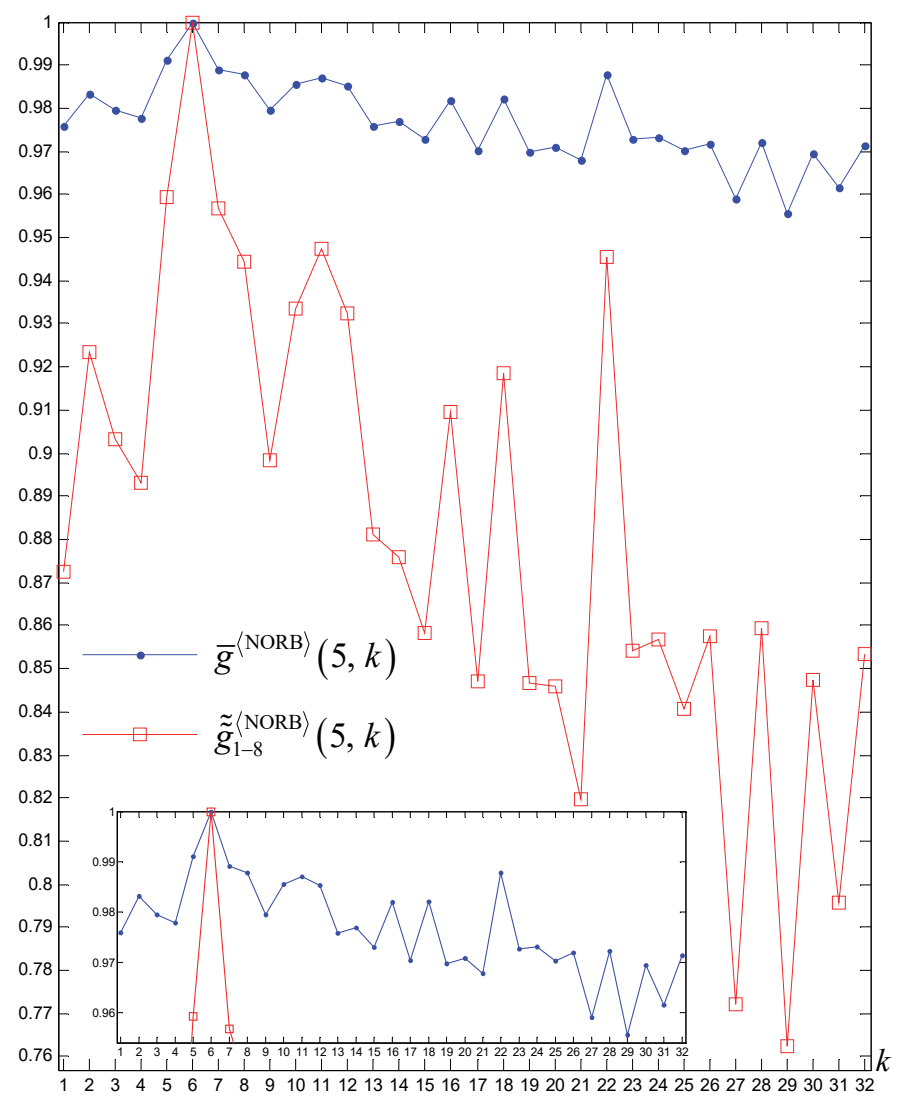

Fig. 17. The normalized averaged RERD (16) and the normalized averaged final-epoch RERD (17) by $N_{\text {ConvL }}=5$ on NORB. Maxima coincide at $k=6$. A weak consistency of these polylines and those ones in Fig. 9 is viewed. 
Figures 14 and 15 show polylines which look pretty opposite and dissimilar to the corresponding polylines plotted for CIFAR-10 and NORB datasets. For CNNs with 4 ConvLs, maximum points do not coincide, and the steepest RERD appears to be at $k=12$ which is the version $1011_{(2)}$ for the successive RERD (16), and at $k=16$ which is the version $1111_{(2)}$ for the final-epoch RERD (17). This is much amazing because the version $1111_{(2)}$ is the worst for EEACL26 dataset under both the averaged performance and final-epoch performance (see Fig. 6). It is probably caused by the fact that the origin of EEACL26 dataset is the simplest, so inserting more DOLs does not produce a negative effect, but only improves generalization. On the other hand, the error rate after the first epoch is much greater for $1111_{(2)}$ (the maximum point in Fig. 14) and $01011_{(2)}, 01110_{(2)}, 01111_{(2)}$ (see these close-to-maximum points in Fig. 15), so the subsequent RERD comes delusively great. For instance, we have obtained

$$
v_{1}^{\langle\text {EEACL26 }\rangle}(32,4,16)=0.3188, v_{8}^{\langle\text {EEACL26 }}(32,4,16)=0.0272,
$$

at $k=16$, but

$$
v_{1}^{\langle\text {EEACL26 }\rangle}(32,4,3)=0.0871, v_{8}^{\langle\text {EEACL26 }\rangle}(32,4,3)=0.0130,
$$

at $k=3$. Therefore, results of RERD like the polylines in Figs. 12-17 should always be checked along with the performance like the polylines in Figs. 4-11.

\section{The Rule of RAtionally Allocating DOLS}

Even using the averaged performance in Figs. 10 and 11, it is hard to determine a single version of DOL allocation which maximises the performance. Moreover, shapes of polylines in Figs. 12, 13, 16, 17 are so contrasting with shapes of polylines in Figs. 14, 15 that averaging over them would be incorrect. This means that some compromise must be reached.

Performances (7), (8), and (13), (12) do depend weakly on the image size along with versions of DOL allocation, but it is impossible to find a rule of this dependence. Dependence on the number of image categories along with the versions is far weaker. But it is very hard to conclude on how performance depends on the complexity of datasets, although such dependence is clearly understandable owing to Figs. 12, 13, 16, 17 and Figs. 14, 15. A rough conclusion is that simpler datasets admit more DOLs (remember those strange maxima of polylines in Figs. 14 and 15).

A connection between the number of ConvLs and an appropriate number of DOLs is unclear. Once again, there is a rough conclusion about that $\mathrm{CNNs}$ with a greater number of ConvLs may have a slightly greater number of DOLs. For compromising with the polylines' contrasts and eccentricity, the most appropriate versions of DOL allocation in CNNs with 4 and 5 ConvLs for CIFAR-10, EEACL26, and NORB datasets are $0010_{(2)}$ and $00010_{(2)}$, respectively. This implies that appropriateness of DOLs and allocation of their 0.5 rates tend to insert, for CNNs with a few ConvLs, just a single DOL before the last ConvL. This rule of rationally allocating DOLs excludes inserting a DOL after every single ConvL, unless the dataset is very simple (like the MNIST dataset [7], [8], [14], [17] resembling EEACL26), where overfitting is likelier. Figure 18 shows error rates for the EEACL26 dataset by using the rule versus using four other versions. Versions of $k=5$ and $k=7$ appear even better. The gain runs up to $50 \%$. For CIFAR-10 the gain is much less (Fig. 19).

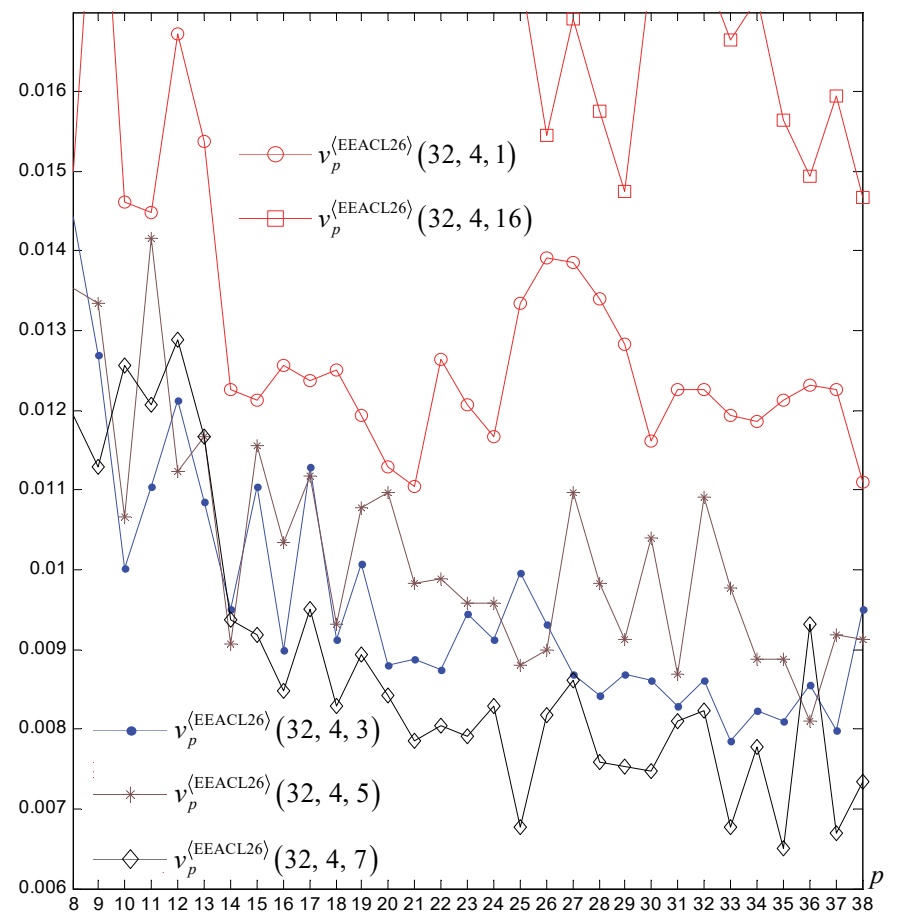

Fig. 18. Error rates against 38 epochs for the EEACL26 dataset. The best gain is achieved at the version of $k=7$, not of $k=3$. The two DOLs seem to win.

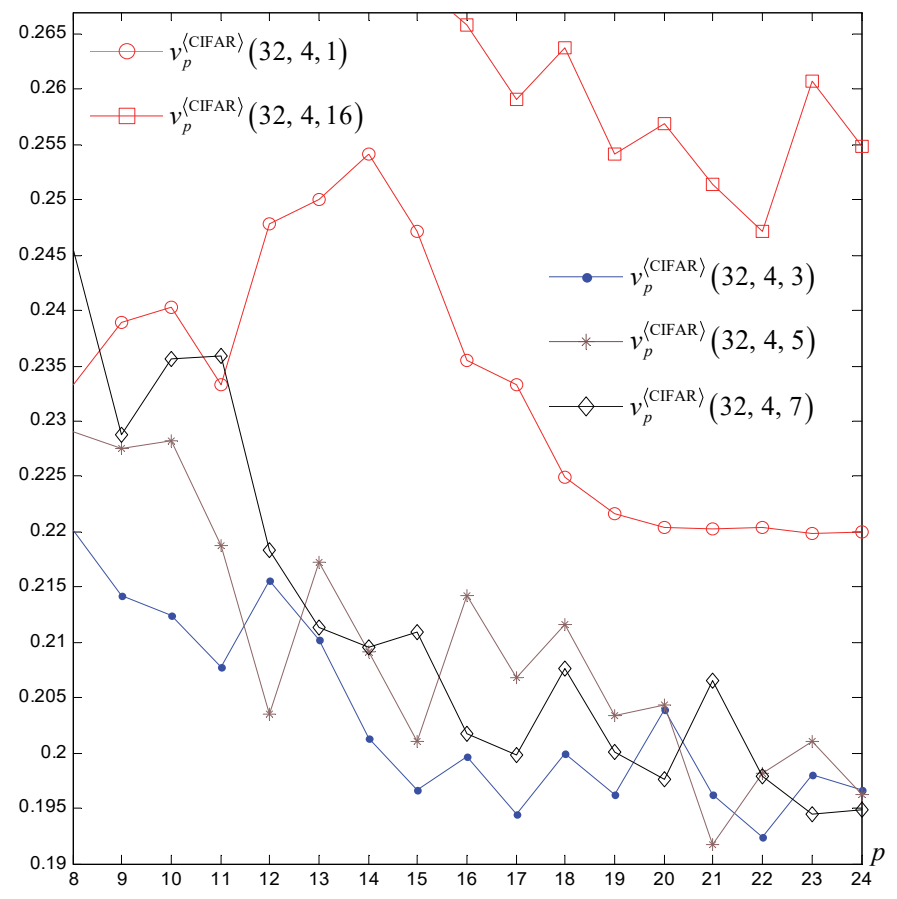

Fig. 19. Error rates against 24 epochs for the CIFAR-10 dataset. Three versions of rational DOL allocation $(3,5,7)$ produce similar accuracy, although duration of training is shorter for the rule's version (a single DOL). 
Inserting two DOLs is not a poor practice, but the DOLs should be as much nearer to the output layer of $\mathrm{CNN}$ as possible. At least, "thin" versions $0101_{(2)}$ and $00101_{(2)}$ do not exceed "compact" versions $0011_{(2)}$ and $00011_{(2)}$. However, versions $0110_{(2)}$ and $00110_{(2)}$ are better than $0011_{(2)}$ and $00011_{(2)}$. This suggests that "compact" versions of allocating DOLs should not be at the output layer. In this way, "thinner" versions, if any, are preferable. The "thinning" is nonetheless recommended to be compact because ultra-thinned versions $1001_{(2)}$ and $10001_{(2)}$ are worse. It confirms that inserting a DOL after the starting ConvLs is a poor practice.

\section{CONCLUSION}

Appropriateness of DOLs and allocation of their 0.5 rates across CNNs consist in non-compactly inserting a few DOLs before the last ConvL. Recommendation of non-compactness is motivated with that compactly bunching up two or more DOLs may cause a bottleneck for information passing through a CNN. To prevent the bottleneck, a single DOL or a few DOLs should be inserted according to either scheme ...010

or, for a greater number of ConvLs (remember Fig. 9 and Fig. 11), scheme $\ldots 01010_{(2)}$, where the ellipsis is substituted with a number of zeros corresponding to the rest of ConvLs. It is likely that the scheme with two or more DOLs fits CNNs of many ConvLs for ICPs with a plenty of features. Such a scheme shall also fit ICPs over simple datasets prone to overfitting.

The said results are trustworthy because CIFAR-10, EEACL26, and NORB datasets used for benchmarking are totally heterogeneous themselves in their origin, internal diversity, number of image categories, coloration, background, hues, illumination, human-eye distinguishability. However, these datasets are insufficient to determine how rational DOL allocation is influenced by the image size and number of image categories. Thus, in further DOL research, the rule for appropriately allocating DOLs shall be expanded regarding directly arguments of the dataset complexity, number of input features, and number of image categories. Besides, the dropout rate is to be optimised starting with that naive 0.5 rate.

\section{REFERENCES}

[1] K. Hagiwara and K. Fukumizu, "Relation Between Weight Size and Degree of Over-Fitting in Neural Network Regression," Neural Networks, vol. 21, no. 1, pp. 48-58, Jan. 2008 https://doi.org/10.1016/j.neunet.2007.11.001

[2] G. E. Hinton, N. Srivastava, A. Krizhevsky, I. Sutskever, and R. R. Salakhutdinov, Improving Neural Networks by Preventing CoAdaptation of Feature Detectors, 2012.

[3] H. Wu and X. Gu, "Towards Dropout Training for Convolutional Neural Networks," Neural Networks, vol. 71, pp. 1-10, Nov. 2015. https://doi.org/10.1016/j.neunet.2015.07.007

[4] A. Iosifidis, A. Tefas, and I. Pitas, "DropELM: Fast Neural Network Regularization with Dropout and DropConnect," Neurocomputing, vol. 162, pp. 57-66, Aug. 2015.

https://doi.org/10.1016/j.neucom.2015.04.006
[5] M. Elleuch, R. Maalej, and M. Kherallah, “A New Design Based-SVM of the CNN Classifier Architecture with Dropout for Offline Arabic Handwritten Recognition," Procedia Computer Science, vol. 80, pp. 1712-1723, 2016. https://doi.org/10.1016/j.procs.2016.05.512

[6] W. Sun and F. Su, "A Novel Companion Objective Function for Regularization of Deep Convolutional Neural Networks," Image and Vision Computing, vol. 60, pp. 58-63, Apr. 2017. https://doi.org/10.1016/i.imavis.2016.11.012

[7] V. V. Romanuke, "Training Data Expansion and Boosting of Convolutional Neural Networks for Reducing the MNIST Dataset Error Rate," Research Bulletin of NTUU "Kyiv Polytechnic Institute", no. 6, pp. 29-34, Dec. 2016. https://doi.org/10.20535/1810-0546.2016.6.84115

[8] N. Srivastava, G. E. Hinton, A. Krizhevsky, I. Sutskever, and R. R. Salakhutdinov, "Dropout: A Simple Way to Prevent Neural Networks from Overfitting," Journal of Machine Learning Research, vol. 15, pp. 1929-1958, 2014.

[9] C. Szegedy, W. Liu, Y. Jia, P. Sermanet, S. Reed, D. Anguelov, D. Erhan, V. Vanhoucke, and A. Rabinovich, "Going Deeper with Convolutions," 2015 IEEE Conference on Computer Vision and Pattern Recognition (CVPR), Jun. 2015. https://doi.org/10.1109/cvpr.2015.7298594

[10] A. Krizhevsky, I. Sutskever, and G. E. Hinton, "ImageNet Classification with Deep Convolutional Neural Networks," Communications of the $A C M$, vol. 60, no. 6, pp. 84-90, May 2017. https://doi.org/10.1145/3065386

[11] K. Simonyan and A. Zisserman, "Very Deep Convolutional Networks for Large-Scale Image Recognition," 5th International Conference on Learning Representations (ICLR 2015), 2015.

[12] J. Kim, O. Sangjun, Y. Kim, and M. Lee, "Convolutional Neural Network with Biologically Inspired Retinal Structure," Procedia Computer Science, vol. 88, pp. 145-154, 2016. https://doi.org/10.1016/j.procs.2016.07.418

[13] D. C. Ciresan, U. Meier, J. Masci, L. M. Gambardella, and J. Schmidhuber, "Flexible, High Performance Convolutional Neural Networks for Image Classification," Proceedings of the Twenty-Second International Joint Conference on Artificial Intelligence, vol. 2, pp. 1237-1242, 2011. https://doi.org/10.5591/978-1-57735-516-8/IJCAI11-210

[14] P. Date, J. A. Hendler, and C. D. Carothers, "Design Index for Deep Neural Networks," Procedia Computer Science, vol. 88, pp. 131-138, 2016. https://doi.org/10.1016/j.procs.2016.07.416

[15] V. V. Romanuke, "Two-Layer Perceptron for Classifying Flat ScaledTurned-Shifted Objects by Additional Feature Distortions in Training," Journal of Uncertain Systems, vol. 9, no. 4, pp. 286-305, 2015.

[16] V. V. Romanuke, "Boosting Ensembles of Heavy Two-Layer Perceptrons for Increasing Classification Accuracy in Recognizing Shifted-Turned-Scaled Flat Images with Binary Features," Journal of Information and Organizational Sciences, vol. 39, no. 1, pp. 75-84, 2015.

[17] E. Kussul and T. Baidyk, "Improved Method of Handwritten Digit Recognition Tested on MNIST Database," Image and Vision Computing, vol. 22, no. 12, pp. 971-981, Oct. 2004. https://doi.org/10.1016/i.imavis.2004.03.008

Vadim V. Romanuke graduated from the Technological University of Podillya (Ukraine) in 2001. In 2006, he received the degree of Candidate of Technical Sciences in Mathematical Modeling and Computational Methods. The degree of Doctor of Technical Sciences in Mathematical Modeling and Computational Methods was received in 2014. In 2016, Vadim Romanuke received the academic status of Full Professor.

$\mathrm{He}$ is a Professor of the Faculty of Navigation and Naval Weapons at the Polish Naval Academy. His current research interests concern decision making, game theory, statistical approximation, and control engineering based on statistical correspondence. He is the author of 302 scientific articles, one monograph, one tutorial, three methodical guidelines in functional analysis, mathematical and computer modelling, conflict-controlled systems. At present, Vadim Romanuke is the scientific supervisor of a Ukrainian budget grant work concerning minimisation of water heat transfer and consumption. $\mathrm{He}$ is also the Head of the Department of Fitting Statistical Approximators at the Centre of Parallel Computations managed by Khmelnitskiy National University.

Address for correspondence: 69 Śmidowicza Street, Gdynia, Poland, 81-127.

E-mail: romanukevadimv@gmail.com 